\title{
MAXELIDE AND ITS DOMAIN OF APPLICATION
}

Dan Parker \& T. Daniel Seely

1. INTRODUCTION: This paper reveals certain false predictions of an otherwise empirically well-motivated condition on ellipsis, Merchant's (2008) MaxElide. Ultimately, we sketch a revision of MaxElide to account for the recalcitrant data, and explore several explanatory benefits of our proposal with potentially interesting implications for our understanding of ellipsis more generally.

2. BACKGROUND: Consider the contrast between Sluicing in (1a) and (wh-)extraction from Verb Phrase Ellipsis (VPE) in (1b).

(1) Sluicing

a. Sue criticized someone, but I don't know who [IP Sue criticized [who]].

$V P E$

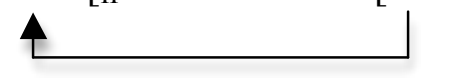

b. *?Sue criticized someone, but I don't know who [IP she did [vp eriticize [whe]]]].

For recent work (see, for example, Fox \& Lasnik (2003)), sluicing is not an independent operation but rather a term used to describe (wh-)movement followed by ellipsis, where ellipsis is the non-expression of phonological information while maintaining the corresponding semantic information. In (1a), the wh-element moved out of its containing IP, followed by ellipsis of the IP. Extraction from ellipsis is thus possible. But, as revealed by Fox \& Lasnik (2003), such extraction is restricted. For example, extraction out of the VP followed by VPE is not always allowed, (1b). The question then emerges: Why the contrast between (1)a and (1)b?

Merchant (2008) proposes a novel constraint MaxElide to account for the ungrammaticality of (1b). The formal statement of MaxElide is given in (2). ${ }^{1}$

(2) MaxElide (Merchant 2008)

Let XP be an elided constituent containing an $\mathrm{A}^{\prime}$-trace

Let $\mathrm{YP}$ be a possible target for deletion

YP must not properly contain XP (XP $\subset /$ YP)

MaxElide predicts that when the relevant licensing conditions are met, sluicing and VPE are in complementary distribution, with the higher/larger ellipsis target IP (sluicing), selected over a lower target, VP (VPE). Thus, consider (3).

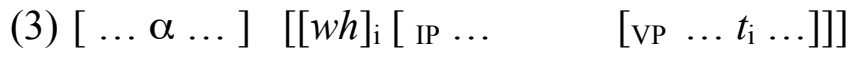
Source
Target-1 Target-2

Assume that $\alpha$ is the correlate of the $w h$-element and that the conditions for the elision of both IP and VP are met. MaxElide, informally stated, requires that the higher target IP (Target-1)

\footnotetext{
${ }^{1}$ See Takahashi \& Fox (2005) for an alternative statement.
} 
is elided and not the lower target VP (Target-2). As Merchant (2008) reveals, this prediction is borne out over a range of cases, (1) a representative illustration.

According to (2), the application of MaxElide is restricted: it applies only in domains containing an $\mathrm{A}^{\prime}$-bound (e.g. wh-) trace. If no $\mathrm{A}^{\prime}$-bound trace exists in the relevant domain (i.e. the target clause), there is the option of elided a higher or lower constituent (where standard conditions for ellipsis are met). Thus, both sluicing and VPE are acceptable in (4).

(4) Ben knows that Sue invited Klaus,
a. ... but her father doesn't.
$=$ sluicing
b. ... but her father doesn't know that she did.
=VPE (Merchant (2008) \#(35))

3. NOT ALL PREDICTIONS BORNE OUT: We report here several crucial cases where the conditions for MaxElide are met, but where sluicing and VPE are not in the predicted complementary distribution. ${ }^{2}$ Instead, both are allowed. (5) - (7) are representative examples $((a)=\text { sluicing, }(b)=V P E)^{3}$

(5) a. Sue criticized some of the students, but I don't know exactly which ONES. and I know exactly which ONES.

b. Sue criticized some of the students, but I don't know exactly which ONES she did. and I know exactly which ONES she did.

(6) a. I know WHAT Sue will read, and I also know WHEN.

b. I know WHAT Sue will read, and I also know WHEN she will.

(7) a. Tyler will read something, but I don't know WHERE.

b. Tyler will read something, but I don't WHERE he will.

There are also cases considered by Schuyler (2001), though not relative to MaxElide (but cf. Takahashi and Fox (2005)).

(8) a. I don't know which PUPPY you should adopt, but I know which KITTEN

b. ?I don't know which PUPPY you should adopt, but I know which KITTEN you should.

MaxElide incorrectly predicts that only sluicing is allowed in (5) - (8).

3. RESTRICTING THE DOMAIN OF APPLICATION OF MAXELIDE: Despite appropriately located $w h$-traces, (5) - (8) pattern after (4) rather than (1). Why is this? Notice, in the well-formed examples, the wh-element is D-linked and stressed, and in the ill-formed cases the $w h$-element is neither D-linked nor stressed. Interestingly, Pesetsky (1987) notes that Dlinked $w h$-elements display unique behavior relative to their neutral, non-D-linked counterparts. For example, Pesetsky illustrates that D-linked $w h$ - in situ does not display Superiority Effects, while the non-D-linked counterpart does, as in (9).

\footnotetext{
${ }^{2}$ See Takahashi \& Fox (2005) and Park (2004) for related examples.

${ }^{3}$ See Hartman (2009) for an independent analysis of $w h$-adverbials.
} 
(9) a. [Which man] id did you persuade $e_{\mathrm{i}}$ to read which book?

cf. who did you persuade to read what?

b. [Which book $]_{\mathrm{I}}$ did you persuade which man to read $e_{\mathrm{i}}$ ?

cf. ??What did you persuade who(m) to read?

(No Superiority)

Pesetsky also notes that $w$ h-phrases such as who and what, while generally non-D-linked, can be given a D-linked interpretation (under the right contextual conditions) "particularly if all the wh-phrases are given extremely heavy stress" (see Pesetsky (1987) p. 108-109). Thus, Superiority is eliminated in (10).

(10) I know what just about everybody was asked to do,
a. ... but WHAT did WHO (actually) do?
(No Superiority)
b. ... but WHAT DID who ACTUALLY do?
(cf. Pesetsky (1987) \#(35))

In a sense, then, for Pesetsky, D-linked wh-in-situ does not leave a "true" wh-trace (under LF movement). There is something "special" about the syntactic behavior of D-linked wh-phrases (beyond their semantic properties, which, as well-known, are different than "regular" whphrases). Suppose we capitalize on Pestesky's insight by formally implementing the idea that a non-D-linked/non-stressed $w$ h-trace in the narrow syntax is a true (non-restricted) variable, and that MaxElide applies only when such a true variable is present. Under this view, D-linked traces form a natural class (not "true" variables), as do unrestricted ("true") variables. Thus, the domain of application is more restricted than assumed in Merchant (2008): it applies only in domains that contain (what would count as) a "true" variable.

3. CONSEQUENCES AND COMPARISIONS: Fox \& Lasnik (2003) provides an alternative account of the core cases in (5) - (8). For F\&L, parallelism is an inviolable constraint (source $=$ target). If there is no successive cyclic movement in the source, there can be none in the target. To account for $w h$-movement in the core cases, F\&L propose that the $w h$-phrase moves in one step from its initial Merge site to its landing site. However, one-step $w h$-movement violates locality constraints. To keep track of the locality constraints, F\&L (tentatively) adopt Chomsky's (1972) "*" notion whereby the island category is marked with *, and the offending island can be "repaired" by ellipsis. If ellipsis fails to delete the *, the expression is rendered unacceptable; thus the contrast in (11):

(11) a. Bob criticized someone but I can't remember who [ $\mathrm{P} *$ Bob criticized [who]].

b. *Bob criticized someone but I can't remember who [IP* he did [vp criticized [whe]]]

Clearly, MaxElide-revised and F\&L overlap in their empirical coverage, and maintaining both analyses would be redundant. Are there any cases that are unique to MaxElide? Potential candidates include ACD constructions (12a) and comparatives (12b), as well as (13) - (14). In these cases, F\&L undergenerate while MaxElide-revised yields the correct results.

(12) a. I met every linguist who you did.

b. You liked some foods more than Tom did. 
(13) What did Tobias read for this class?
a. I don't know.
b. ?I don't know what.
c. *?I don't know what he did.

(14) Bill criticized some of the students, but I don't know which ONES he did.

Does MaxElide (revised) subsume the F\&L analysis? Not entirely, given that MaxElide undergenerates in (15) while F\&L get the right result.

(15) Sluice (repairs island)

a. They hired someone who speaks a Balkan language but I don't remember which [language they hired someone whospeaks]

VPE (does not repair island, yet MaxElide is satisfied)

b. *They DID hire someone who speaks a Balkan language but I don't remember what kind of language they DIDN'T [hire someone who speaks what kind of language]

To account for (16b), Merchant (2008) employs a (form of a) crucial component of the F\&L analysis, namely the island marking * device, (16). Thus, MaxElide-revised equipped with an island marking device yields the necessary data coverage.

$$
[\ldots \alpha \ldots] \quad\left[[w h]_{\mathrm{i}}\left[\mathrm{IP} / \mathrm{VP} \ldots \quad\left[\mathrm{xP}^{*} \ldots t_{\mathrm{i}} \ldots\right]\right]\right] \quad(\text { sluice }=\mathrm{IP}, \mathrm{VPE}=\mathrm{VP})
$$

Overall, we restrict the application of MaxElide, in what we argue to be a natural way, with results that maintain all the advantages of MaxElide (as presented by Merchant), while simultaneously extending its empirical coverage.

\section{References:}

Fox, D. and H. Lasnik. 2003. Successive cyclic movement and island repair: The difference between Sluicing and VP Ellipsis. Linguistic Inquiry 34:143-154.

Hartman, Jeremy. (to appear). The Semantic Effects of non-A' Traces: Evidence from Ellipsis Parallelism. Proceedings of SALT 19.

Lasnik, Howard. 2001. When can you save a structure by destroying it? In M. Kim and U. Strauss (eds.) Proceedings of NELS 31, GSLA Publications.

Merchant, Jason. 2008. Variable island repair under ellipsis. In K. Johnson (ed.), Topics in ellipsis, Cambridge University Press: Cambridge.

Pesetsky, David. 1987. WH-in-situ: Movement and Unselective Binding. In E. Reuland and A.G.B. terMeulen (eds.), The Linguistic Representation of (In)definiteness, MIT Press, Cambridge.

Schuyler, Tamara. 2001. Wh-movement out of the site of VP-ellipsis. MA thesis, Department of Linguistics, University of California Santa Cruz. In Syntax and Semantics at Santa Cruz, Séamas MacBhloscaidh (ed). LRCPublications, Santa Cruz, California

Takahashi, S. \& Fox, D. 2005. MaxElide and the Re-binding Problem. In E. Georgala \& J Howell (eds.) Proceedings of SALT XV, CLC Publications. 